\title{
A lower bound for coherences on the Brown-Peterson spectrum
}

\author{
BIRGIT RICHTER
}

\begin{abstract}
We provide a lower bound for the coherence of the homotopy commutativity of the Brown-Peterson spectrum, $B P$, at a given prime $p$ and prove that it is at least $\left(2 p^{2}+2 p-2\right)$-homotopy commutative. We give a proof based on Dyer-Lashof operations that $B P$ cannot be a Thom spectrum associated to $n$-fold loop maps to $B S F$ for $n=4$ at 2 and $n=2 p+4$ at odd primes. Other examples where we obtain estimates for coherence are the Johnson-Wilson spectra, localized away from the maximal ideal and unlocalized. We close with a negative result on Morava- $K$-theory.
\end{abstract}

55P43; $13 \mathrm{D} 03$

\section{Introduction}

Recently obstruction theory for imposing $E_{\infty}$-structures on homotopy commutative and associative ring spectra has been successfully applied in some cases of very wellbehaved higher chromatic spectra like $E_{n}$ (Goerss-Hopkins [12], Richter-Robinson [23]) and $\widehat{E(n)}$ (Baker-Richter [3]) and up to chromatic type one [3].

The two approaches for such an obstruction theory that are available at the moment were developed by Paul Goerss and Mike Hopkins [12] on the one hand and Alan Robinson [24] on the other hand. They use André-Quillen type cohomology theories as the habitat for possible obstructions which turn out to be equivalent (see Basterra-Richter [5, Theorem 2.6]). The crucial point in all the examples mentioned above is that one can rely on an étaleness property of the algebra of cooperations in order to make all obstruction groups vanish.

It is an old, still open question raised by Peter May, whether the Brown-Peterson spectrum, $B P$, is an $E_{\infty}$ ring spectrum. There have been serious attempts to prove that $B P$ has an $E_{\infty}$ model. In fact, topological André-Quillen homology defined by Maria Basterra in [4] was originally introduced to solve this problem (compare Kriz [16]).

Unlike complex cobordism, $M U$, or other Thom spectra, $B P$ is not born with an $E_{\infty}-$ structure. Therefore trying obstruction theory methods seems to be a canonical attempt. But the algebra of cooperations $B P_{*} B P$, is a polynomial algebra on countably many 
generators, and this will produce a lot of non-trivial André-Quillen type cohomology groups.

However, the possible obstruction groups for instance in the setting of Robinson's Gamma cohomology only occur in a small range of degrees. The approach of this note is to exploit the sparseness of these degrees together with the sparseness of the coefficients

$$
B P_{*}=\mathbb{Z}_{(p)}\left[v_{1}, v_{2}, \ldots\right], \text { with }\left|v_{i}\right|=2 p^{i}-2
$$

and the algebra $B P_{*} B P$ to obtain an estimate for the coherence of the homotopy commutativity of $B P$. We admit that this lower bound might be much too pessimistic, but it seems that there is some interest in even partial results.

Maria Basterra and Mike Mandell announced that $B P$ has at least an $E_{4}$-structure, ie, possesses an action by the little 4-cubes operad. By results of Fiedorowicz-Vogt [10] or Basterra-Mandell this implies that the topological Hochschild homology spectrum of $B P, T H H(B P)$, is at least an $E_{3}$-spectrum and the natural map $B P \rightarrow T H H(B P)$ is a map of $E_{3}-$ spectra.

Our approximation result depends on the prime involved.

Theorem 1.1 The Brown-Peterson spectrum BP at a prime $p$ has at least a $\left(2 p^{2}+\right.$ $2 p-2)$-stage structure.

Here, an $n$-stage structure is a certain filtration step towards an $E_{\infty}$-structure. Alan Robinson introduced this filtration in [24] and we will describe it in Section 3. We will prove Theorem 1.1 in Section 6.2.

The obstruction theory which we will use is the one developed by Alan Robinson [24; 25]. We will explain it in detail in sections 2-4.

At the moment we do not have a direct comparison between $n$-stage structures and structures over some version of the little cubes operad.

However, $n$-stage structures are of independent interest because they give rise to Dyer-Lashof operations in a certain range of degrees (see Section 7). In the case of $B P$ one could get homology operations by comparing the $\mathbb{F}_{p}$-homology of $B P$ with the one of $M U$ and $H \mathbb{F}_{p}$ (see Bruner-May-McClure-Steinberger [6, page 63]). We use Dyer-Lashof operations to show in Theorem 7.2 that $B P$ cannot be the Thom spectrum associated to an $n$-fold loop map to $B S F$. Here $n$ is four for the even prime and $2 p+4$ for any odd prime $p$. We stress that a stronger result is mentioned in Lewis' thesis [18]. 
In other examples the existence of Dyer-Lashof operations might help for instance with calculations of topological Hochschild homology.

The main object of study of this note is the Brown-Peterson spectrum. The reader who is primarly interested in this example might skip sections $2-4$ and might procede directly to Section 5. The material presented in sections 2-4 is needed for cases like the Johnson-Wilson spectra. We discuss this and other examples in Section 8.

Acknowledgements I thank Sverre Lunøe-Nilsen and John Rognes for their interest. They pointed out to me that my partial coherence results should lead to Dyer-Lashof operations. Thanks to Andy Baker who read earlier versions of this and made important comments. I am grateful to John Rognes and the Department of Mathematics in Oslo for their hospitality. Parts of this work were written when I was in Bonn and I would like to thank Carl-Friedrich Bödigheimer for his constant support during the last years. The author was partially supported by the Strategisk Universitetsprogram i Ren Matematikk (SUPREMA) of the Norwegian Research Council.

\section{Some background on Gamma (co)homology}

Let $k$ be a (graded) commutative ring with unit, let $A$ be a (graded) commutative $k$-algebra and let $M$ be a (graded) $A$-module. In the following tensor products will all be taken with respect to $k$. Gamma homology of $A$ over $k$ with coefficients in $M$ is defined in Robinson [24, 2.5] as the homology of the total complex of a bicomplex $\Xi_{*, *}$ which we will now describe.

Let $\operatorname{Lie}(n)$ be the $n^{\text {th }}$ term of the operad which codifies Lie algebras over $k$, ie, Lie $(n)$ is the free $k$-module generated by all Lie monomials in variables $x_{1}, \ldots, x_{n}$ such that each variable appears exactly once. There is a canonical action of the symmetric group on $n$ letters, $\Sigma_{n}$, on $\operatorname{Lie}(n)$ by permuting the variables $x_{i}$. Let $\operatorname{Lie}(n)^{*}$ be the $k$-linear dual of $\operatorname{Lie}(n)$. Then the bicomplex for Gamma homology in bidegree $(r, s)$ is defined as

$$
\Xi_{r, s}(A \mid k ; M)=\operatorname{Lie}(s+1)^{*} \otimes k\left[\Sigma_{s+1}\right]^{\otimes r} \otimes A^{\otimes(s+1)} \otimes M .
$$

Here, all undecorated tensor products are taken over the ground ring $k$. The horizontal differential is the differential of the bar construction; the vertical differential is complicated and we refer the curious reader to [24, Secion 2] for details. For our purpose it is enough to know that it induces multiplication of the algebra entries, induces an action of $A$ on $M$ and does something with the permutations in $\Sigma_{s+1}$ to reduce them to elements in $\Sigma_{S}$. 
We emphasize that all these operations preserve the internal degree if $A$ and $M$ are graded. Therefore it makes sense to define the $i^{\text {th }}$ homogeneous part of $\Xi_{*, *}$ and the associated total complex. Gamma homology for graded commutative algebras therefore possesses a natural bigrading, where $\mathrm{H} \Gamma_{q, i}(A \mid k ; M)$ is the $q^{\text {th }}$ homology of the $i^{\text {th }}$ homogeneous part of the total complex of $\Xi_{*, *}(A \mid k ; M)$. Gamma cohomology, which is defined via the homomorphism complex out of the total complex of $\Xi_{*, *}(A \mid k ; A)$ into $M$, inherits the internal grading. Following [24] we denote by $\operatorname{H} \Gamma^{q, i}(A \mid k ; M)$ the $q^{\text {th }}$ cohomology of the homomorphism complex

$$
\operatorname{Hom}_{A}^{i}\left(\operatorname{Tot}\left(\Xi_{*, *}(A \mid k ; A)\right), M\right)
$$

whose morphisms lower degree by $i$.

\section{Robinson's obstruction theory}

Consider the product of the topological version of the Barratt-Eccles operad $\left(E \Sigma_{n}\right)_{n}$ and Boardman's tree operad $\left(T_{n}\right)_{n}$. Here the space of $n$-trees, $T_{n}$, consists of abstract trees on $n+1$ leaves. These leaves are labelled with the numbers $0, \ldots, n$ where each label appears exactly once. Internal edges get an assigned length $0<\lambda \leqslant 1$. This tree space is set to consist of a point for $n \leqslant 2$, the only 2 -tree is the tree

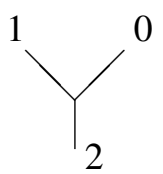

There are three different types of 3-trees, namely
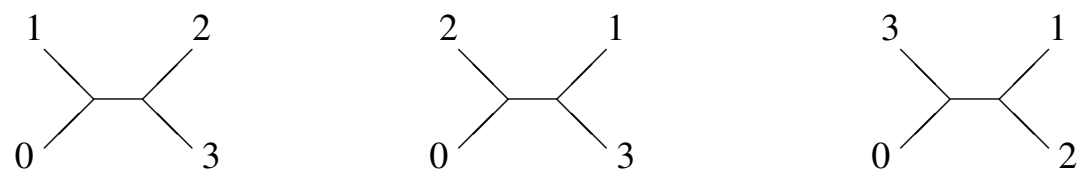

with a corolla-shaped tree if the length of the only internal edge is shrunk to zero.

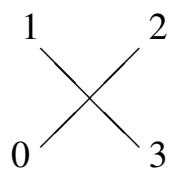

For arbitrary $n$, the space $T_{n}$ is contractible with the corolla on $n+1$ leaves as basepoint. Composition in the tree operad is given by grafting trees. The newly built internal edge in the composed tree is defined to be of length one. The $n^{\text {th }}$ part of the operad which Robinson uses is the product operad

$$
\mathcal{B}_{n}=E \Sigma_{n} \times T_{n} .
$$


As $E \Sigma_{n}$ is $\Sigma_{n}$-free and contractible and as $T_{n}$ is contractible, the product operad $\mathcal{B}$ is an $E_{\infty}$-operad. Robinson defines a filtration of this $E_{\infty}$-operad as follows: set $\mathcal{B}_{n}^{(i)}:=\left(E \Sigma_{n}\right)^{(i)} \times T_{n}$ where $\left(E \Sigma_{n}\right)^{(i)}$ is the $i^{\text {th }}$ skeleton of the standard model for $E \Sigma_{n}$. Then define

$$
\nabla^{n} \mathcal{B}_{m}:=\mathcal{B}_{m}^{(n-m)}
$$

Definition 3.1 $[24,5.3]$ An $n$-stage structure for an $E_{\infty}$-structure on a spectrum $E$ is a sequence of maps

$$
\mu_{m}: \nabla^{n} \mathcal{B}_{m} \ltimes_{\Sigma_{m}} E^{\wedge m} \longrightarrow E
$$

which on their restricted domain of definition satisfy the requirements for an operad action on $E$.

Let us make explicit what that amounts to in small filtration degrees. A 2-stage structure on a spectrum $E$ consists of action maps starting from $\nabla^{2} \mathcal{B}_{m}$ which is

$$
\mathcal{B}_{m}^{(2-m)}=\left(E \Sigma_{m}\right)^{(2-m)} \times T_{m}
$$

The $(2-m)$-skeleton of $E \Sigma_{m}$ is trivial for $m>2$, therefore the only requirement is that we have a map

$$
\left(\left(E \Sigma_{1}\right)^{(1)} \times T_{1}\right) \ltimes_{\Sigma_{1}} E \cong E \stackrel{\varphi}{\longrightarrow} E
$$

and that $E$ possesses a map

$$
\left(\left(E \Sigma_{2}\right)^{(0)} \times T_{2}\right) \ltimes_{\Sigma_{2}} E^{\wedge 2} \cong\left(\Sigma_{2}\right) \ltimes_{\Sigma_{2}} E^{\wedge 2} \longrightarrow E .
$$

This is nothing but a multiplication $\mu$ on $E$ together with its twisted version $\mu \circ \tau$ if $\tau$ denotes the generator of $\Sigma_{2}$. The axioms of an operad action force the map $\varphi$ to be the identity of $E$. Iterates of $\mu$ and $\mu \circ \tau$ act on higher smash powers of $E$, but they do not have to satisfy any relations.

A 3-stage structure on $E$ comes with three kinds of maps, because non-trivial values for $m$ are 1,2,3. The first value does not give anything new. The second step requires action maps

$$
\left(\left(E \Sigma_{2}\right)^{(1)} \times T_{2}\right) \ltimes_{\Sigma_{2}} E^{\wedge 2} \longrightarrow E .
$$

The 1-skeleton of $E \Sigma_{2}$ is the 1-circle, giving the homotopy between $\mu$ and $\mu \circ \tau$.

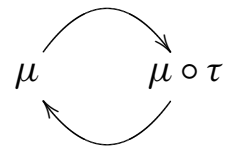

Algebraic $8 \mathcal{G}$ Geometric Topology, Volume 6 (2006) 
In addition to that, the value $m=3$ brings in the homotopies for associativity via the trees we described above, because we obtain maps

$$
\left(\left(E \Sigma_{3}\right)^{(0)} \times T_{3}\right) \ltimes_{\Sigma_{3}} E^{\wedge 3} \cong\left(\Sigma_{3} \times T_{3}\right) \ltimes_{\Sigma_{3}} E^{\wedge 3} \longrightarrow E .
$$

Theorem 3.2 [24, Theorem 5.5] Assume that $E$ is a homotopy commutative and associative ring spectrum which satisfies

$$
E^{*}\left(E^{\wedge m}\right) \cong \operatorname{Hom}_{E_{*}}\left(E_{*} E^{\otimes m}, E_{*}\right) \text { for all } m \geqslant 1 .
$$

If $E$ has an $(n-1)$-stage structure which can be extended to an $n$-stage structure then possible obstructions to extending this further to an $(n+1)$-stage structure live in

$$
\mathrm{H} \Gamma^{n, 2-n}\left(E_{*} E \mid E_{*} ; E_{*}\right) .
$$

If in addition $\mathrm{H} \Gamma^{n, 1-n}\left(E_{*} E \mid E_{*} ; E_{*}\right)$ vanishes, then this extension is unique.

We start with a 3 -stage structure. If we want to establish an $n$-stage structure on $E$, then we have to show that Gamma cohomology vanishes in bidegrees $(\ell, 2-\ell)$ for all $n-1 \geqslant \ell \geqslant 3$. (This is incorrectly stated in $[24,5.6]$ but tacitly corrected in $[25,5.8]$.)

Let us note that $B P$ satisfies the necessary properties to apply Robinson's obstruction theory: $B P$ is a homotopy commutative $M U$-ring spectrum at all primes, Strickland $[27,2.8,2.9]$, and it satisfies the requirement from (2).

\section{Where the obstructions come from}

In this section we will explain the geometric origin of Robinson's obstruction groups [24]. We do not present anything new here, but focus on some details that we will need later in Section 8.

The obstruction groups in the theorem above arise from a geometric object: one can extend an $n$-stage structure to an $(n+1)$-stage structure if certain cohomology classes vanish. An $n$-stage always gives rise to maps out of parts of $\nabla^{n+1} \mathcal{B}_{m}$ as well, because we require that the action maps $\mu_{m}$ satisfy the axioms of operad actions where they are defined. Therefore these actions are closed under composition. Robinson calls the part in $\nabla^{n+1} \mathcal{B}_{m}$ that is generated by compositions the boundary of $\nabla^{n+1} \mathcal{B}_{m}$ and denotes it by $\partial \nabla^{n+1} \mathcal{B}_{m}$. Let $Q_{n+1}^{m}$ be the cofibre of the inclusion of $\partial \nabla^{n+1} \mathcal{B}_{m} \cup \nabla^{n} \mathcal{B}_{m}$ into 
$\nabla^{n+1} \mathcal{B}_{m}$ and consider the following diagram:

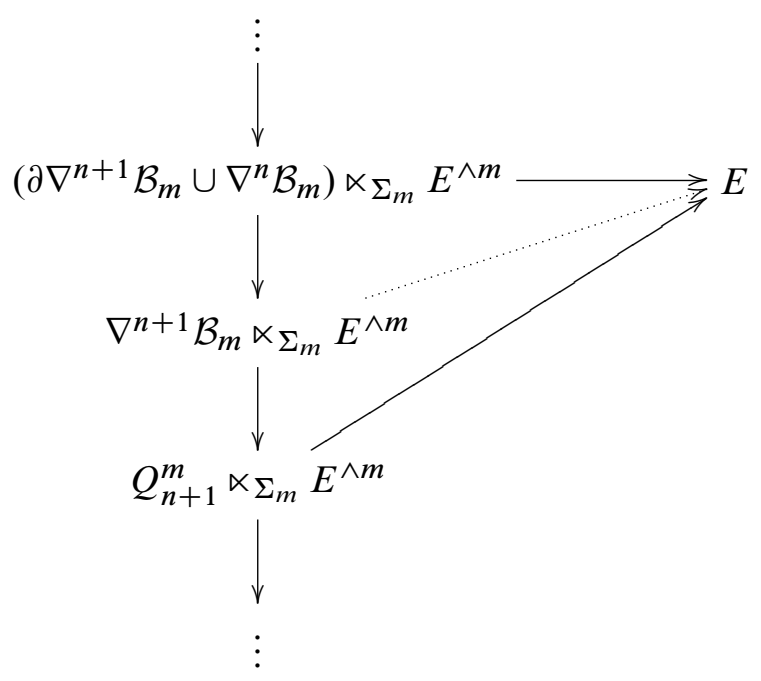

We obtain a long exact sequence in $E$-cohomology

$$
\begin{aligned}
\ldots \rightarrow E^{0}( & \left.Q_{n+1}^{m} \ltimes_{\Sigma_{m}} E^{\wedge m}\right) \rightarrow E^{0}\left(\nabla^{n+1} \mathcal{B}_{m} \ltimes_{\Sigma_{m}} E^{\wedge m}\right) \rightarrow \\
& \rightarrow E^{0}\left(\left(\partial \nabla^{n+1} \mathcal{B}_{m} \cup \nabla^{n} \mathcal{B}_{m}\right) \ltimes_{\Sigma_{m}} E^{\wedge m}\right) \rightarrow E^{1}\left(Q_{n+1}^{m} \ltimes_{\Sigma_{m}} E^{\wedge m}\right) \rightarrow \ldots
\end{aligned}
$$

If the first $E$-cohomology group of $Q_{n+1}^{m} \ltimes_{\Sigma_{m}} E^{\wedge m}$ vanishes, then one can extend the action map to the $(n+1)^{\text {st }}$ filtration step. If the term $E^{0}\left(Q_{n+1}^{m} \ltimes_{\Sigma_{m}} E^{\wedge m}\right)$ is trivial as well, then the extension of the $n$-stage structure to an $(n+1)$-stage structure is unique. Note, that we have to consider all $2 \leqslant m \leqslant n+1$, because these are the non-trivial domains for an $(n+1)$-stage action.

If one has a universal coefficient theorem at hand, one can then continue to identify this cohomology group as $\operatorname{Hom}_{E_{*}}\left(E_{*}\left(Q_{n+1}^{m} \ltimes_{\Sigma_{m}} E^{\wedge m}\right), E_{*}\right)$. Note, that up to this stage homomorphisms are taken with respect to $E_{*}$ and not $E_{*} E$. Alan Robinson then identifies $E_{*}\left(Q_{n+1}^{m} \ltimes \Sigma_{m} E^{\wedge m}\right)$ as some tractable algebraic object and induces homomorphism up to $E_{*} E$. Furthermore he proves in [24, Proposition 5.4] that obstruction are always cocycles for a suitable coboundary map, which then gives the final identification with Gamma cohomology groups.

However, one could stop at earlier stages before passing to the level of Gamma cohomology groups. One can try to extract direct information either out of

$$
E^{1}\left(Q_{n+1}^{m} \ltimes_{\Sigma_{m}} E^{\wedge m}\right)
$$

Algebraic 83 Geometric Topology, Volume 6 (2006) 
or, in the presence of a universal coefficient theorem for $E$, one can investigate in which degrees the groups

$$
\operatorname{Hom}_{E_{*}}^{1}\left(E_{*}\left(Q_{n+1}^{m} \ltimes_{\Sigma_{m}} E^{\wedge m}\right), E_{*}\right)
$$

are non-trivial.

In the examples that we will consider, we already know that the spectra under consideration possess $A_{\infty}$-structures. Therefore we have universal coefficient spectral sequences available converging to the cohomological term (3).

Let us examine the $E$-homology of $Q_{n+1}^{m} \ltimes_{\Sigma_{m}} E^{\wedge m}$ further in order to get control over internal degree shifts.

We use Robinson's geometric identification of $Q_{n+1}^{m}$ as $E \Sigma_{m}^{(n-m+1)} / E \Sigma_{m}^{(n-m)} \wedge$ $T_{m} / \partial T_{m}$. Here the second quotient is the quotient of the cubical tree complex modulo the subcomplex of all fully grown trees - these are decomposable trees, ie, trees with at least one internal edge having length one. The first quotient is equivalent to $\bigvee_{\Sigma_{m}^{n-m+2}} \mathbb{S}^{n-m+1}$ whereas the identification in [24] and Whitehouse [28] shows that the second quotient has the homotopy type of $\bigvee_{(m-1) !} \mathbb{S}^{m-2}$ which on the level of homology gives rise to the dual of the Lie representation. One copy of $\Sigma_{m}$ is swallowed by the $\ltimes_{\Sigma_{m}}$. The degree shift caused by spheres adds up to a total of $n-1$, so in $E$-homology we get the term

$$
\Sigma^{n-1} \operatorname{Lie}(m)^{*} \otimes E_{*}\left[\Sigma_{m}\right]^{\otimes(n-m+1)} \otimes E_{*} E^{\otimes m} .
$$

Therefore the $E_{*}$-homomorphisms on these are given as

$$
\begin{aligned}
\operatorname{Hom}_{E_{*}}^{1}\left(E _ { * } \left(Q_{n+1}^{m} \ltimes \Sigma_{m}\right.\right. & \left.\left.E^{\wedge m}\right), E_{*}\right) \\
& \cong \operatorname{Hom}_{E_{*}}^{2-n}\left(\operatorname{Lie}(m)^{*} \otimes E_{*}\left[\Sigma_{m}\right]^{\otimes(n-m+1)} \otimes E_{*}\left(E^{\wedge m}\right), E_{*}\right) .
\end{aligned}
$$

\section{The case of $B P$}

Potential obstruction classes for an extension to an $(n+1)$-stage structure on $B P$ live in bidegree $(n, 2-n)$. Here $n$ is the cohomological degree and $2-n$ is the internal degree. The algebra of cooperations $B P_{*} B P$ over $B P_{*}$ is a polynomial ring over $B P_{*}$,

$$
B P_{*} B P=B P_{*}\left[t_{1}, t_{2}, \ldots\right] \text { with }\left|t_{i}\right|=2 p^{i}-2 .
$$


We are therefore in the situation where we can apply the universal coefficient theorem to see that the obstruction groups arise from

$$
\begin{aligned}
\operatorname{Hom}_{B P_{*}}^{1}\left(B P _ { * } \left(Q_{n+1}^{m}\right.\right. & \left.\left.\ltimes \Sigma_{m} B P^{\wedge m}\right), B P_{*}\right) \\
& \cong \operatorname{Hom}_{B P_{*}}^{2-n}\left(\operatorname{Lie}(m)^{*} \otimes B P_{*}\left[\Sigma_{m}\right]^{\otimes(n-m+1)} \otimes B P_{*} B P^{\otimes m}, B P_{*}\right) .
\end{aligned}
$$

In order to achieve an internal degree of the form $2-n$, we have to look for a map that raises degree by $n-2$. Everything in sight is concentrated in degrees of the form

$$
i=\sum_{i=1}^{N} \lambda_{j}\left(2 p^{j}-2\right)
$$

where the $\lambda_{j}$ are non-negative integers. Any non-trivial map

$$
\operatorname{Lie}(m)^{*} \otimes B P_{*}\left[\Sigma_{m}\right]^{\otimes(n-m+1)} \otimes B P_{*} B P^{\otimes m} \rightarrow B P_{*}
$$

can only alter the degree again by a degree of the form $i=\sum \lambda_{j}\left(2 p^{j}-2\right)$, because $B P_{*}$ is concentrated in the same degrees. The minimal such degree bigger than zero is $2 p-2$.

Obstruction groups occur as Gamma cohomology of bidegree $(n, 2-n)$ for $n \geqslant 3$. Therefore the first possibly non-trivial obstruction group could live in $\mathrm{H}^{2 p, 2-2 p}$, which could contain an obstruction to extending a $2 p$-stage structure to a $(2 p+1)-$ stage structure. This amounts to saying that $B P$ has at least a $2 p$-stage structure.

\section{Refined estimates}

The previous section dealt with an argument which was merely a degree count. We will improve the $2 p$-estimate by having a detailed look at the actual homological level. We consider the universal coefficient spectral sequence for Gamma cohomology of $B P_{*} B P$. Its $E_{2}$-term is of the form

$$
\operatorname{Ext}_{B P_{*} B P}^{*, *}\left(\mathrm{H} \Gamma_{*, *}\left(B P_{*} B P \mid B P_{*} ; B P_{*} B P\right), B P_{*}\right) \Rightarrow \mathrm{H} \Gamma^{*, *}\left(B P_{*} B P \mid B P_{*} ; B P_{*}\right) .
$$

Remark 6.1 In the spectral sequence (5) the cohomological degree $s$ for our possible obstruction group $\mathrm{H} \Gamma^{s, 2-s}$ is now spread over the groups

$$
\begin{aligned}
& \operatorname{Ext}_{B P_{*} B P}^{0, s}\left(\mathrm{H} \Gamma_{*, *}\left(B P_{*} B P \mid B P_{*} ; B P_{*} B P\right), B P_{*}\right), \\
& \ldots, \mathrm{Ext}_{B P_{*} B P}^{s, 0}\left(\mathrm{H} \Gamma_{*, *}\left(B P_{*} B P \mid B P_{*} ; B P_{*} B P\right), B P_{*}\right)
\end{aligned}
$$

so we have to prove, that all these groups vanish. As we grade homologically the internal degree $j$ in $\mathrm{Ext}^{i, j}$ corresponds to a map that lowers degree by $j$. For instance 
a homomorphism $f$ in $\operatorname{Hom}_{B P_{*} B P}^{j}\left(\mathrm{H} \Gamma_{*, *}\left(B P_{*} B P \mid B P_{*} ; B P_{*} B P\right), B P_{*}\right)$ is a sequence of maps

$$
f=\left(f_{s}\right) ; \quad f_{s}: H \Gamma_{s, *}\left(B P_{*} B P \mid B P_{*} ; B P_{*} B P\right) \longrightarrow B P_{s-j}
$$

The reformulations in the rest of this section will not preserve the internal grading of Gamma homology. The following result is an immediate application of RobinsonWhitehouse [26, 6.8(2)].

Lemma 6.2 Gamma homology groups for $B P_{*} B P=B P_{*}\left[t_{1}, t_{2}, \ldots\right]$ split into Gamma homology groups for the single pieces $B P_{*}\left[t_{i}\right]$ :

$$
\mathrm{H} \Gamma_{s, *}\left(B P_{*} B P \mid B P_{*} ; B P_{*} B P\right) \cong \bigoplus_{i \geqslant 1} \mathrm{H} \Gamma_{s, *}\left(B P_{*}\left[t_{i}\right] \mid B P_{*} ; B P_{*} B P\right) .
$$

We start with simplifying each summand in the splitting (6).

Lemma 6.3 Gamma homology of $B P_{*}\left[t_{i}\right]$ can be expressed as follows:

$$
\begin{aligned}
\mathrm{H} \Gamma_{s, *}\left(B P_{*}\left[t_{i}\right] \mid B P_{*} ; B P_{*} B P\right) \\
\\
\cong B P_{*} \otimes_{\mathbb{Z}_{(p)}} \mathrm{H} \Gamma_{s, *}\left(\mathbb{Z}_{(p)}\left[t_{i}\right] \mid \mathbb{Z}_{(p)} ; \mathbb{Z}_{(p)}\right) \otimes_{\mathbb{Z}_{(p)}} B P_{*} B P .
\end{aligned}
$$

Proof For Gamma homology there is a flat-base-change result (see [26, 6.8 (1)]) which ensures that

$$
\mathrm{H} \Gamma_{s, *}\left(B P_{*}\left[t_{i}\right] \mid B P_{*} ; B P_{*} B P\right) \cong B P_{*} \otimes_{\mathbb{Z}_{(p)}} \mathrm{H} \Gamma_{s, *}\left(\mathbb{Z}_{(p)}\left[t_{i}\right] \mid \mathbb{Z}(p) ; B P_{*} B P\right) .
$$

Gamma homology of a polynomial generator is ignorant of the taken coefficients: the Steenrod splitting of $[23,4.1]$ identifies $H \Gamma_{s, *}\left(\mathbb{Z}_{(p)}\left[t_{i}\right] \mid \mathbb{Z}_{(p)} ; B P_{*} B P\right)$ with Gamma homology with trivial coefficients induced up to $B P_{*} B P$

$$
\mathrm{H} \Gamma_{s, *}\left(\mathbb{Z}_{(p)}\left[t_{i}\right] \mid \mathbb{Z}_{(p)} ; B P_{*} B P\right) \cong \mathrm{H} \Gamma_{s, *}\left(\mathbb{Z}_{(p)}\left[t_{i}\right] \mid \mathbb{Z}_{(p)} ; \mathbb{Z}_{(p)}\right) \otimes B P_{*} B P
$$

In [23] we proved this in the ungraded case. Gamma homology of a graded algebra has homogeneous components and the above isomorphism has no reason to preserve the internal grading.

Gamma homology of a polynomial algebra in a single variable with coefficients in the ground ring was identified in [23, Proposition 3.2].

Lemma 6.4 Summing over all internal degrees, we can identify Gamma homology of $\mathbb{Z}_{(p)}\left[t_{i}\right]$ as

$$
\bigoplus_{t \geqslant 0} \mathrm{H} \Gamma_{s, t}\left(\mathbb{Z}_{(p)}\left[t_{i}\right] \mid \mathbb{Z}_{(p)} ; \mathbb{Z}_{(p)}\right) \cong\left(H \mathbb{Z}_{(p)}\right)_{s} H \mathbb{Z}
$$


Proof Using [23] and the identification of Gamma homology with stable homotopy groups of $\Gamma$-modules from Pirashvili-Richter [22] we obtain

$$
\mathrm{H} \Gamma_{s, t}\left(\mathbb{Z}_{(p)}\left[t_{i}\right] \mid \mathbb{Z}_{(p)} ; \mathbb{Z}_{(p)}\right) \cong \begin{cases}\pi_{s}^{s t}\left(\mathcal{L}_{j}\left(\mathbb{Z}_{(p)}\left[t_{i}\right] \mid \mathbb{Z}_{(p)} ; \mathbb{Z}_{(p)}\right)\right) & \text { if } j\left(2 p^{i}-2\right)=t \\ 0 & \text { otherwise. }\end{cases}
$$

where $\mathcal{L}_{j}\left(\mathbb{Z}_{(p)}\left[t_{i}\right] \mid \mathbb{Z}_{(p)} ; \mathbb{Z}_{(p)}\right)$ is the $j^{\text {th }}$ homogeneous component of an appropriate $\Gamma$-module. Taking all degrees together gives the claim because in [23, Theorem 4.1] the homotopy group $\pi_{s}^{s t}\left(\mathcal{L}\left(\mathbb{Z}_{(p)}\left[t_{i}\right] \mid \mathbb{Z}_{(p)} ; \mathbb{Z}_{(p)}\right)\right)$ has been identified with $\left(H \mathbb{Z}_{(p)}\right)_{s} H \mathbb{Z}$.

In positive degrees $\left(H \mathbb{Z}_{(p)}\right)_{*} H \mathbb{Z}$ is just the $p$-torsion part of $H \mathbb{Z}_{*} H \mathbb{Z}$. Kochman computed this explicitly in [15, page 44$]$.

\subsection{Kochman's description of $\left(H \mathbb{Z}_{(p)}\right)_{*} H \mathbb{Z}$}

Kochman provides in [15, Theorem 3.5 (c)] an explicit basis of the $p$-torsion in $H \mathbb{Z}_{*} H \mathbb{Z}$. The result is:

- There is only simple $p$-torsion.

- An explicit basis of $\left(H \mathbb{Z}_{(p)}\right)_{*} H \mathbb{Z}$ over $\mathbb{Z} / p \mathbb{Z}$ consists of all expressions

$$
P\left(n_{1}, \ldots, n_{t}\right) \bar{\zeta}_{1}^{e_{1}} \cdot \ldots \cdot \bar{\zeta}_{s}^{e_{s}}
$$

where $t \geqslant 0, t \neq 1,0<n_{1}<\ldots<n_{t}, e_{i} \geqslant 0, t+e_{1}+\ldots+e_{s}>0$ and $e_{i}=0$ for $i<n_{1}$. Here, the degree of the $P\left(n_{1}, \ldots, n_{t}\right)$ is $2\left(p^{n_{1}}+\ldots+p^{n_{t}}\right)-t-1$ and the degree of $\bar{\zeta}_{i}$ is $2\left(p^{i}-1\right)$ with the convention that the degree of $P()=1$ is zero for $t=0$.

Important for us are the cases $t=0$ and $t=2$. For $t=0$ the condition $e_{i}=0$ for $i<n_{1}$ is void, therefore elements like $\bar{\zeta}_{1}^{e_{1}} \ldots \bar{\zeta}_{s}^{e_{s}}$ arise with at least one $e_{i}$ being positive. These elements have total degree

$$
\operatorname{degree}\left(\bar{\zeta}_{1}^{e_{1}} \cdot \ldots \cdot \bar{\zeta}_{s}^{e_{s}}\right)=\sum_{i=1}^{s} e_{i}\left(2 p^{i}-2\right)
$$

For $t=2$ the situation is a little bit more involved because non-trivial factors like $P(n, m)$ occur. The element of lowest possible degree in this case is $P(1,2)$ with

$$
\text { degree }(P(1,2))=2 p+2 p^{2}-2-1=2 p^{2}+2 p-3 .
$$




\subsection{Proof of Theorem 1.1}

Obstructions for extending an $n$-stage to an $(n+1)$-stage structure live in $\mathrm{H} \Gamma^{n, 2-n}$. From our previous arguments we know that $\left(H \mathbb{Z}_{(p)}\right)_{*} H \mathbb{Z}$ corresponds to $\mathrm{H} \Gamma_{*, *}\left(\mathbb{Z}_{(p)}\left[t_{i}\right] \mid \mathbb{Z}_{(p)} ; \mathbb{Z}_{(p)}\right)$ and consists only of simple $p$-torsion, ie, summands of $\mathbb{Z} / p \mathbb{Z}$ as we are working in $\mathbb{Z}_{(p)}$-modules. For Gamma homology of $B P_{*} B P$, which is given by

$$
\bigoplus_{i \geqslant 0} B P_{*} \otimes_{\mathbb{Z}_{(p)}} \mathrm{H} \Gamma_{*, *}\left(\mathbb{Z}_{(p)}\left[t_{i}\right] \mid \mathbb{Z}_{(p)} ; \mathbb{Z}_{(p)}\right) \otimes_{\mathbb{Z}_{(p)}} B P_{*} B P
$$

we can therefore find a free $B P_{*} B P$-resolution of length one given by direct sums of shifted copies of $B P_{*} B P \stackrel{p}{\longrightarrow} B P_{*} B P$.

We therefore obtain that it suffices to consider $\mathrm{Ext}^{0, *}-$ and $\mathrm{Ext}^{1, *}$-terms in the Universal Coefficient spectral sequence (5). We know as well, that the internal degree can only be of the form $\sum_{i=1}^{N} \lambda_{i}\left(2 p^{i}-2\right)$; consequently possible values for $n$ have to be of the form $\sum_{i=1}^{N} \lambda_{i}\left(2 p^{i}-2\right)+2$ with the $\lambda_{i}$ being non-negative integers.

We are only concerned with positive homological degrees, therefore the corresponding Gamma homology groups will be torsion. As there are no non-trivial homomorphism from $B P_{*} B P / p$ to $B P_{*}$ the groups

$$
\operatorname{Ext}_{B P_{*} B P}^{0, n}\left(\bigoplus_{i \geqslant 0} B P_{*} \otimes_{\mathbb{Z}_{(p)}} \mathrm{H} \Gamma_{*, *}\left(\mathbb{Z}_{(p)}\left[t_{i}\right] \mid \mathbb{Z}_{(p)} ; \mathbb{Z}_{(p)}\right) \otimes_{\mathbb{Z}_{(p)}} B P_{*} B P, B P_{*}\right)
$$

all vanish.

Thus we are looking for the smallest possible degree of a non-vanishing

$$
\operatorname{Ext}_{B P_{*} B P}^{1, n-1}\left(\bigoplus_{i \geqslant 0} B P_{*} \otimes_{\mathbb{Z}_{(p)}} \mathrm{H} \Gamma_{*, *}\left(\mathbb{Z}_{(p)}\left[t_{i}\right] \mid \mathbb{Z}_{(p)} ; \mathbb{Z}_{(p)}\right) \otimes_{\mathbb{Z}_{(p)}} B P_{*} B P, B P_{*}\right)
$$

As $B P_{*}$ is concentrated in non-negative degrees, a non-trivial map which lowers degree by $n-1$ can come from Gamma homology in homological degree $n-1+$ $\sum_{k=1}^{N^{\prime}} \mu_{k}\left(2 p^{k}-2\right)$. Using the constraint for $n$ gotten above this degree can hit non-trivial groups only for

$$
n-1+\sum_{k=1}^{N^{\prime}} \mu_{k}\left(2 p^{k}-2\right)=\sum_{i=1}^{N} \lambda_{i}\left(2 p^{i}-2\right)+1+\sum_{k=1}^{N^{\prime}} \mu_{k}\left(2 p^{k}-2\right) .
$$


Considering the degrees of Kochman's generators $P\left(n_{1}, \ldots, n_{t}\right) \bar{\zeta}_{1}^{e_{1}} \cdot \ldots \cdot \bar{\zeta}_{s}^{e_{s}}$ in the case $t=0$ gives the equation

$$
\sum_{i=1}^{N} \lambda_{i}\left(2 p^{i}-2\right)+1+\sum_{k=1}^{N^{\prime}} \mu_{k}\left(2 p^{k}-2\right)=\sum_{j=1}^{M} e_{j}\left(2 p^{j}-2\right),
$$

so 1 had to be even and we all believe that this is a contradiction.

The next lowest degrees are to be found for $t=2$. We content ourselves with considering a possible cohomology class of degree $n-1$. Here the corresponding equation of degrees that has to be satisfied is

$$
\sum_{i=1}^{N} \lambda_{i}\left(2 p^{i}-2\right)+1=2 p^{n}+2 p^{m}-3+\sum_{j=1}^{M} e_{j}\left(2 p^{j}-2\right) .
$$

The generator $P(1,2)$ is of lowest possible degree and turns this requirement into

$$
n-1=\sum_{i=1}^{N} \lambda_{i}\left(2 p^{i}-2\right)+1=2 p+2 p^{2}-3=2 p-2+2 p^{2}-2+1 .
$$

Therefore such a homology class could occur for $n=2 p^{2}+2 p-2$.

Remark 6.5 Of course, the bound in Theorem 1.1 is just an estimate. As some of the identification did not preserve the internal degree, this obstruction group may be zero. Even if it is non-trivial, it does not have to contain an actual obstruction. Many people believe that $B P$ is in fact an $E_{\infty}$ ring spectrum, and our proof does not disprove that.

The vanishing of Gamma cohomology for degree reasons in a certain range has a second important consequence. Even if there is a homotopy commutative multiplication that obeys higher coherences, it is an issue whether this structure is unique or whether there are different ways to find homotopies which care for the higher coherences. Note, that the $M U_{*}$-algebra structure of $B P_{*}$ gives $B P$ a unique $M U$-ring structure [13, 2.21] and this in turn gives rise to a homotopy commutative and associative ring structure on $B P$, ie, a 3-stage structure for $B P$. We get the following estimate.

Corollary 6.6 Any 3-stage structure for BP extends uniquely to a $(2 p-1)$-stage structure.

Proof Obstruction groups for the unique extension of an $n$-stage structure to an $(n+1)$-stage structure occur as $\mathrm{H} \Gamma^{n, 1-n}\left(B P_{*} B P \mid B P_{*} ; B P_{*}\right)$. The internal degree $1-n$ has to be of the form $-\sum_{i=1}^{M} \lambda_{i}\left(2 p^{i}-2\right)$, therefore we obtain the constraint 
$n=\sum_{i=1}^{M} \lambda_{i}\left(2 p^{i}-2\right)+1$. For the same reasons as before homological degree $n$ corresponds to an $\mathrm{Ext}^{0, *}$-term and vanishes. So we have to obtain an estimate for the lowest possible $(n-1)^{\text {st }}$ Gamma homology group. Here, the cases for $t=0$ turn out to be relevant. The equation for a possible degree is

$$
n-1=\sum_{i=1}^{M} \lambda_{i}\left(2 p^{i}-2\right)=\sum_{i=1}^{N} e_{i}\left(2 p^{i}-2\right) .
$$

This can occur for $e_{1}=1$ and $e_{i}=0$ for $i>0$, so we obtain $n-1=2 p-2$. Consequently $\mathrm{H} \Gamma^{2 p-1,2-2 p}\left(B P_{*} B P \mid B P_{*} ; B P_{*}\right)$ might be non-trivial, and hence a given $(2 p-1)-$ stage structure with a fixed $(2 p-2)$-stage structure might be prolonged to a $2 p$-stage structure, but in probably different ways.

Remark 6.7 Note, that this estimate for uniqueness is not better than the one we would have achieved by a mere degree counting argument. We will obtain similar results later for the localized Johnson-Wilson spectra $\mathcal{E}(n)$.

\section{Dyer-Lashof operations}

An $n$-stage structure on a spectrum $E$ gives rise to some low-degree Dyer-Lashof operations. If $E$ were an $H_{\infty}$-spectrum (see [6]) then the $i^{\text {th }}$ Dyer-Lashof operation $Q_{i}$ is defined as follows: take the standard resolution, traditionally called $W$ in that context, of the cyclic group of order $p, \mathbb{Z} / p \mathbb{Z}$, and assume without loss of generality that your spectrum comes with a $C W$-structure which is compatible with the $H_{\infty}-$ structure. Let $C_{*}$ denote cellular chain functor for spectra. Then we obtain a map $\vartheta$ as follows.

$\vartheta: W \otimes_{\mathbb{Z} / p \mathbb{Z}} C_{*}(E)^{\otimes p} \rightarrow C_{*}\left(E \Sigma_{p}\right) \otimes_{\Sigma_{p}} C_{*}(E)^{\otimes p} \cong C_{*}\left(E \Sigma_{p} \ltimes_{\Sigma_{p}} E^{\wedge p}\right) \rightarrow C_{*}(E)$.

In this situation one can define $Q_{i}(x)$ for $x \in H \mathbb{F}_{p_{*}}(E)$ as

$$
Q_{i}(x):=\vartheta_{*}\left(e_{i} \otimes x^{\otimes p}\right) .
$$

Here $e_{i}$ is the generator of $W_{i}$. Note that this just uses the $i$-skeleton of $E \Sigma_{p}$; but this is part of an $n$-stage structure on $E$ for $i \leqslant n-p$. Therefore we get the following result.

Proposition 7.1 If $E$ has an $n$-stage structure with $n>p$ then there are Dyer-Lashof operations $Q_{i}$ on the $\mathbb{F}_{p}$-homology of $E$ for $i \leqslant n-p$. 
The usual Dyer-Lashof operations $Q^{i}$ are then given by regrading as

$$
Q^{i}(x)=\left\{\begin{array}{llll}
0 & \text { if } i<|x| & \text { and } & p=2 \\
Q_{i-|x|}(x) & \text { if } i \geqslant|x| & \text { and } & p=2 \\
0 & \text { if } 2 i<|x| & \text { and } & p>2 \\
\pm Q_{(2 i-|x|)(p-1)}(x) & \text { if } 2 i \geqslant|x| & \text { and } & p>2
\end{array}\right.
$$

Andy Baker noticed a curious fact about Dyer-Lashof operations on $B P$. First, let $p$ be an odd prime. The indecomposable element $a_{p-1} \in\left(H \mathbb{F}_{p}\right)_{2 p-2}(M U)$ is known to be in the image of $\left(H \mathbb{F}_{p}\right)_{2 p-2}(B P)$. Consider an element $x=x_{2 p-2}$ in $\left(H \mathbb{F}_{p}\right)_{2 p-2}(B P)$ with image $a_{p-1}$. For such an $x$ the highest Dyer-Lashof operation $Q^{i}$ which we get out of the $\left(2 p^{2}+2 p-2\right)$-stage structure is $Q^{2 p}$. In $[14,2.11]$ $\mathrm{Hu}$, Kriz and May proved that the inclusion from $B P$ to $M U$ cannot be a map of commutative $S$-algebras, and they used this particular Dyer-Lashof operation to show that (compare the correction of the proof of $[14,2.11]$ in $[2$, Appendix B]). The image of $a_{p-1}$ under $Q^{2 p}$ is $a_{(2 p+1)(p-1)}$ up to decomposable elements, but there is no indecomposable element in $\left(H \mathbb{F}_{p}\right)_{(2 p+1)(p-1)}(B P)$. For $p=2$ a similar argument works using $a_{1}$.

In the following $S F$ denotes the colimit $S F=\operatorname{colim} S F_{n}$ where $S F_{n}$ is the monoid of based homotopy equivalences of $\mathbb{S}^{n}$ of degree one, so $B S F$ is the classifying space of spherical fibrations. We emphasize that the following result is not optimal. In his thesis Lewis [18, pages 145-6] wrote down a sketch of an argument due to Priddy, that $B P$ cannot be the $p$-localization of a Thom spectrum associated to a map of $H$-spaces from a double loop space $X$ to $B S F$. Priddy's argument involves the Eilenberg-Moore spectral sequence and calculations with secondary cohomology operations.

I thank Stewart Priddy and Yuli Rudyak for telling me that Lewis' thesis still is the only written account of that argument. We offer our weaker result here because we think that the proof via Dyer-Lashof operations which we give here is short and straightforward.

Theorem 7.2 The Brown-Peterson spectrum BP cannot be the $p$-localization of a Thom spectrum associated to a 4 -fold loop map to $B S F$ at $p=2$ resp. a $(2 p+4)-$ fold loop map to $B S F$ at any odd prime $p$.

Proof Assume there were such a map from an $n$-fold loop space $X$ to $B S F$

$$
\gamma: X \longrightarrow B S F
$$

which would allow to write $B P$ as the Thom spectrum associated to $\gamma, B P=X^{\gamma}$. Lewis' result states that Thom spectra associated to $n$-fold loop maps to $B S F$ are 
$E_{n}$-spectra [19, Theorem IX.7.1]. Here $E_{n}$ is the product of the little- $n$-cubes operad and the linear isometries operad.

The Thom isomorphism tells us that the homology of $B P$ is isomorphic to the homology of $X$, and the latter maps to the homology of $B S F$. The isomorphism respects the Dyer-Lashof operations [19, Proposition IX.7.4 (i)]. In the following $H$ will denote homology with $\mathbb{F}_{p}$-coefficients.

If $p=2$, the homology of $B S F$ is

$$
H_{*}(B S F) \cong H_{*}(B S O) \otimes C_{*},
$$

whereas at odd primes, the homology of $B S F$ is isomorphic to

$$
H_{*}(B S F) \cong H_{*} W \otimes C_{*}^{\prime} .
$$

Explicit formulæ for $C_{*}$ and $C_{*}^{\prime}$ can be found in [9, page 114]. The map from $B S O$ to $B S F$ is an infinite loop map and it is this map which includes the tensor factor $H_{*}(B S O)$ into $H_{*}(B S F)$. Therefore the tensor factor $H_{*}(B S O)$ is closed under the Dyer-Lashof operations. A similar remark applies to $W$ which is a summand of $B O$ at odd primes, because there is a splitting of infinite loop spaces

$$
B O_{(p)} \simeq W \times W^{\perp}
$$

for any odd prime $p$. In particular, for $x=x_{2 p-2}$ in $H_{*}(B P)$ with $\mathcal{P}_{*}^{1}(x)=1$ we obtain a non-trivial class of degree $2 p-2$ in $H_{*}(B S F)$. From [9, pages 114-5] it is clear that there is no class of that degree in the $C_{*}-$ resp. $C_{*}^{\prime}$-part of the above tensor product, and therefore $x$ has to have an image in $H_{*}(B S O)$ resp. $H_{*}(W)$. In both cases, $x$ has to hit an indecomposable element, whose $Q^{2 p}$-image gives a generator up to decomposable elements. The lack of indecomposables in $H_{*}(B P)$ yields a contradiction.

Example 7.3 One can ask whether our approach yields any new Dyer-Lashof operations for the topological Hochschild homology of the Brown-Peterson spectrum. An $E_{3}$-structure on $T H H(B P)$ justifies the calculation of $T H H(B P)$ given in [21, pages 23-4] as

$$
T H H(B P)_{*} \cong B P_{*} \otimes \Lambda\left(\lambda_{1}, \lambda_{2}, \ldots\right), \quad \text { degree }\left(\lambda_{i}\right)=2 p^{i}-1 .
$$

See [7, Section 6] as well.

A pure degree estimate or even a more detailed analysis than in the proof of Theorem 1.1 gives a possible obstruction class in cohomological degree $\mathrm{H} \Gamma^{2 p, 2-2 p}$ caused by $\lambda_{1}$. Therefore $T H H(B P)$ has at least a $2 p$-stage structure for all primes. In terms of 
Dyer-Lashof operations this gives $Q^{i}(x)$ for $(2 i-|x|)(p-1) \leqslant p$, thus $2 i-|x|<2$ at odd primes and for $(i-|x|) \leqslant 2$ at 2 . An $E_{3}$-structure would provide operations $Q^{i}$ on $x$ for $2 i-|x|<2$ at odd primes and $i-|x|<2$ at 2 (compare [6, III.3.1]).

\section{Browder operations}

The geometry of $n$-stage structures gives rise to additional homology operations, which give homological obstructions to extending an $n$-stage structure to an $(n+1)$-stage structure. Recall that an $n$-stage structure includes actions by operad subspaces which come from operadic composition of lower stages. For instance an operad product

$$
\gamma:\left(E \Sigma_{2}^{(0)} \times T_{2}\right) \times\left(E \Sigma_{i}^{(0)} \times T_{i}\right) \times\left(E \Sigma_{j}^{(0)} \times T_{j}\right) \rightarrow\left(E \Sigma_{i+j-1}^{(0)} \times T_{i+j-1}\right)
$$

gives elements in $\Sigma_{n+1} \times \partial T_{n+1}$ for $i+j-1=n+1$ which act on $E$ if the ring spectrum $E$ admits an $n$-stage structure where $\partial T_{n+1} \subset T_{n+1}$ is the subspace of fully-grown $(n+1)$-trees. We already used the identification of the homology of the quotient $T_{n+1} / \partial T_{n+1}$ as the dual of the Lie representation. As $T_{n+1}$ is contractible, the $H \mathbb{F}_{p}$-homology of $\partial T_{n+1}$ is isomorphic to $\left(H \mathbb{F}_{p}\right)_{*+1}\left(T_{n+1} / \partial T_{n+1}\right)$. On the homological level we obtain therefore an action of this shifted copy of $\operatorname{Lie}(n+1)^{*}$ :

$$
\begin{aligned}
\Theta_{*}:\left(H \mathbb{F}_{p}\right)_{*}\left(\Sigma_{n+1}\right. & \left.\times \partial T_{n+1}\right) \otimes \Sigma_{n+1}\left(H \mathbb{F}_{p}\right)_{*}(E)^{\otimes n+1} \\
& \cong\left(H \mathbb{F}_{p}\right)_{*}\left(\partial T_{n+1}\right) \otimes\left(H \mathbb{F}_{p}\right)_{*}(E)^{\otimes n+1} \longrightarrow\left(H \mathbb{F}_{p}\right)_{*}(E) .
\end{aligned}
$$

If the $n$-stage structure on $E$ could be refined to an $(n+1)$-stage structure, then this operation has to be trivial, because it factors over the entire tree space $T_{n+1}$ which is contractible.

Cohen's Theorem 12.3 in [9] expresses the action of the top-dimensional class of the $k$-configurations in $\mathbb{R}^{m}, F\left(\mathbb{R}^{m}, k\right)$, with an iterated Browder operation. The homology of configuration spaces in turn is related to free Lie algebras. In [9, pages 263-4] resp. [8, Theorem 6.1] Cohen describes the homology of $F\left(\mathbb{R}^{m}, k\right)$. For an even $m>0$,

$$
H_{(k-1)(m-1)}\left(F\left(\mathbb{R}^{m}, k\right) ; \mathbb{Z}\right) \cong \operatorname{Lie}(k)
$$

as a $\mathbb{Z}\left[\Sigma_{k}\right]$-module ${ }^{1}$. Up to suspension and a process of dualization, this is precisely the homology of the space of fully grown $(n+1)$-trees, for $k=n+1$.

In the context of iterated loop spaces, a non-trivial Browder operation $\lambda_{n}$ (coming from an $(n+1)$-fold loop space structure on a space) is an obstruction to extending

\footnotetext{
${ }^{1}$ Note that Cohen considers $\operatorname{Lie}(k)$ without the sign-representation [8, page 32] whereas Robinson twists the $\Sigma_{k}$-action on $\operatorname{Lie}(k)$ by the sign [24, page 333]. Therefore we have to adjust Cohen's statement to the above form.
} 
this structure to an $(n+2)$-fold loop space structure. The geometric operation from (10) relates this to obstructions for $(n+1)$-stage structures.

It would be of interest to clarify some of the open questions about possible $E_{\infty}-$ structures on spectra by finding non-trivial operations as in (10). We hope that someone who is more skillful with calculations than the author might actually succeed to find obstruction classes.

\section{Other examples}

In the following we discuss examples where we obtain estimates for homotopy coherence by a mere degree count, either because the actual (co)homology calculation would be hard if not impossible or because we come across a cohomology class for which we have good reasons to believe that it is an actual obstruction class. The localized Johnson-Wilson spectra are examples of the first type whereas the Morava- $K$-theories are examples of the second kind.

\subsection{Localized Johnson-Wilson $\mathcal{E}(i)$}

We start by considering the localized version of the Johnson-Wilson spectra $E(i), \mathcal{E}(i)$ with $\mathcal{E}(i)_{*} \cong\left(B P\langle i\rangle_{*}\right)_{I_{i}}$ (compare [1]). The coefficients of $\mathcal{E}(i)_{*}$ are the coefficients of $B P\langle i\rangle$ localized away from the ideal $I_{i}=\left(p, v_{1}, \ldots, v_{i-1}\right)$. Therefore units in $\mathcal{E}(i)_{*}$ are of the form $s v_{i}^{j}+r$ with $s$ a unit in the $p$-local integers, $j \in \mathbb{Z}$ and $r \in I_{i}$ (compare [1, Section 1]). By [1] it is known that $\mathcal{E}(i)_{*} \mathcal{E}(i)$ is free over $\mathcal{E}(i)_{*}$. Thus possible obstructions live in

$$
\operatorname{Hom}_{\mathcal{E}(i)_{*}}^{2-n}\left(\operatorname{Lie}(m)^{*} \otimes \mathcal{E}(i)_{*}\left[\Sigma_{m}^{n-m+1}\right] \otimes \mathcal{E}(i)_{*} \mathcal{E}(i)^{\otimes m}, \mathcal{E}(i)_{*}\right) .
$$

As $\mathcal{E}(i)$ is Landweber exact over $B P$, the algebra of cooperations can be described as

$$
\mathcal{E}(i)_{*} \mathcal{E}(i)=\mathcal{E}(i)_{*} \otimes_{B P_{*}} B P_{*} B P \otimes_{B P_{*}} \mathcal{E}(i)_{*} .
$$

The degrees of this algebra and hence the degrees of the $\mathcal{E}(i)_{*}$-module

$$
\operatorname{Lie}(m)^{*} \otimes \mathcal{E}(i)_{*}\left[\Sigma_{m}^{n-m+1}\right] \otimes \mathcal{E}(i)_{*} \mathcal{E}(i)^{\otimes m}
$$

are concentrated in degrees of the form

$$
\sum_{\substack{i=1 \\ i \neq n}}^{M} \lambda_{i}\left(2 p^{i}-2\right)+\mu_{n}\left(2 p^{n}-2\right)
$$

with the $\lambda_{i}$ being non-negative integers and $\mu_{n} \in \mathbb{Z}$.

Algebraic ${ }^{6} \mathcal{G}$ Geometric Topology, Volume 6 (2006) 
As this is the underlying module for the chain complex whose dual gives Gamma cohomology we obtain the following result.

Proposition 8.1 The $i^{\text {th }}$ localized Johnson-Wilson spectrum $\mathcal{E}(i)$ possesses at least a $2 p$-stage structure which is unique up to the $(2 p-1)$-stage.

Proof For any potential obstruction we need a non-trivial $\mathcal{E}(i)_{*}$-linear morphism which raises degree by some number of the form $n-2$ with $n \geqslant 3$. As the domain $\operatorname{Lie}(m)^{*} \otimes \mathcal{E}(i)_{*}\left[\sum_{m}^{n-m+1}\right] \otimes \mathcal{E}(i)_{*} \mathcal{E}(i)^{\otimes m}$ and the target $\mathcal{E}(i)_{*}$ are concentrated in degrees as calculated in (11) the minimal such difference of degrees is $2 p$. Therefore there might be an obstruction to extending a given $2 p$-stage structure to a $(2 p+1)-$ stage structure.

\subsection{Ordinary Johnson-Wilson spectra}

The $i^{\text {th }}$ Johnson-Wilson spectrum $E(i)$ is Landweber exact and a homotopy commutative ring spectrum for all primes. The algebra of cooperations $E(i)_{*} E(i)$ is flat over $E(i)_{*}$ and hence the Künneth theorem applies, but we have to use the universal coefficient spectral sequence to control the source $E(i)^{1}\left(Q_{n+1}^{m} \ltimes_{\Sigma_{m}} E(i)^{\wedge m}\right)$ of possible obstructions for highly coherent homotopy commutative structures. The $E_{2}$-term of this spectral sequence is as follows:

$$
\begin{aligned}
\operatorname{Ext}_{E(i)_{*}}^{p, q} & \left(E(i)_{*}\left(Q_{n+1}^{m} \ltimes_{\Sigma_{m}} E(i)^{\wedge m}\right), E(i)_{*}\right) \\
& \cong \operatorname{Ext}_{E(i)_{*}}^{p, q}\left(\Sigma^{n-1} \operatorname{Lie}(m)^{*} \otimes E(i)_{*}\left[\Sigma_{m}\right]^{\otimes(n-m+1)} \otimes E(i)_{*} E(i)^{\otimes m}, E(i)_{*}\right)
\end{aligned}
$$

Here the $(n-1)^{\text {st }}$ suspension in $E(i)$-homology comes from the geometry of the filtration quotient $Q_{n+1}^{m}$ as it was discussed in Section 4.

In this spectral sequence we just have to consider the 0 - and 1-line, because $E(i)_{*} E(i)$ is a countable colimit of free $E(i)_{*}$-modules and the other tensor factors are free. As we are only interested in cohomological degree one and $\operatorname{Ext}_{E(i)_{*}}^{p, q}$ corresponds to cohomological degree $p+q$ we have to determine, for which $n \geqslant 3$ there are non-trivial $\operatorname{Ext}_{E(i)_{*}}^{0,1}-$ and $\operatorname{Ext}_{E(i)_{*}}^{1,0}-$ terms. Taking the internal $(n-1)$-shift into account we have to look out for homomorphisms which raise degree by $n-2$ resp. $n-1$. Counting degrees once again we obtain the following result.

Proposition 8.2 The $i^{\text {th }} J o h n s o n-W i l s o n ~ s p e c t r u m ~ E(i)$ possesses at least a $(2 p-1)-$ stage structure which is unique up to the $(2 p-2)$-stage. 
Proof Both sides are concentrated in degrees of the form

$$
\sum_{\substack{i=1 \\ i \neq n}}^{M} \lambda_{i}\left(2 p^{i}-2\right)+\mu_{n}\left(2 p^{n}-2\right) .
$$

Due to the possibility of a non-trivial Ext ${ }^{1,1-n}$-term, the lowest possible value for $n$ is $n=2 p-2+1=2 p-1$.

Remark 8.3 Note that the above estimate is much too weak for $i=1$ because $E(1)$ possesses a unique $E_{\infty}$-structure at all primes [3, Theorem 6.2].

\subsection{Morava- $K$-theory}

Last but not least we will close with a negative result. It is known that none of the Morava- $K$-theories could possess $E_{\infty}$-structures: if $K(n)$ had one, then its connective cover $k(n)$ had one as well; in particular, $k(n)$ would be an $H_{\infty}$-spectrum which then had to split as a wedge of suspensions of Eilenberg-MacLane spectra (see [6, III, Theorem 4.1]). The argument in [6, III, Theorem 4.1] even shows that $k(n)$ cannot possess any $\mathrm{H}_{2}$-structure. From [20, 2.10] it follows that the connected cover functor $c$ [20, VII.3.2] sends $H_{2}$-spectra to $H_{2}$-spectra. Therefore $K(n)$ cannot have an $E_{2}$-structure, because this would give rise to such an $H_{2}$-structure on $k(n)$.

At $p=2, K(n)$ is even not homotopy commutative. For $M U_{(p)}$-algebra structures on $K(n)$ see $[11 ; 17]$. At odd primes, the $K(n)$ are homotopy commutative and associative ring spectra.

Consider $K(n)$ for an odd prime $p$. We know that the algebra of cooperations $K(n)_{*}(K(n))$ for $K(n)$ consists of an étale part tensored with an exterior algebra $\Lambda_{K(n)_{*}}\left(\tau_{0}, \tau_{1}, \ldots, \tau_{n-1}\right)$ with the $\tau_{i}$ being elements of degree $2 p^{i}-1$. This non-étale part could give rise to possibly non-trivial obstructions. Here $\tau_{0}$ is of internal degree one. A possible non-trivial obstruction groups could therefore be obtained by a class in cohomological degree $n=2+1=3$. Hence even at odd primes there might be a non-trivial obstruction to extending the homotopy commutative structure on $K(n)$ to a 4-stage structure.

Remark 8.4 The situation for the spectrum $P(n)$ at odd primes with $P(n)_{*}=$ $\mathbb{F}_{p}\left[v_{n}, v_{n+1}, \ldots\right]$ is the same as the one for Morava- $K$-theory. This spectrum cannot possess an $\mathrm{H}_{2}$-structure. The algebra of cooperations is

$$
P(n)_{*} P(n)=P(n)_{*} \otimes_{B P_{*}} B P_{*} B P \otimes \Lambda\left(a_{0}, \ldots, a_{n-1}\right)
$$

with $a_{i}$ being of degree $2 p^{i}-1$; in particular $a_{0}$ has degree one, which should give rise to an obstruction to a $4-$ stage structure.

Algebraic $8 \mathcal{G}$ Geometric Topology, Volume 6 (2006) 
In both cases, $K(n)$ and $P(n)$, there are candidates for non-trivial Browder operations for $K(n)$-homology respectively $H \mathbb{F}_{p}$-homology, but we have no proof so far that these homology classes actually arise in this way.

\section{References}

[1] A Baker, $I_{n}$-local Johnson-Wilson spectra and their Hopf algebroids, Doc. Math. 5 (2000) 351-364 MR1767568

[2] A J Baker, JP May, Minimal atomic complexes, Topology 43 (2004) 645-665 MR2041635

[3] A Baker, B Richter, On the $\Gamma$-cohomology of rings of numerical polynomials and $E_{\infty}$ structures on $K$-theory, Comment. Math. Helv. 80 (2005) 691-723 MR2182697

[4] M Basterra, André-Quillen cohomology of commutative S-algebras, J. Pure Appl. Algebra 144 (1999) 111-143 MR1732625

[5] M Basterra, B Richter, (Co-)homology theories for commutative ( $S$-)algebras, from: "Structured ring spectra", London Math. Soc. Lecture Note Ser. 315, Cambridge Univ. Press, Cambridge (2004) 115-131 MR2122156

[6] R R Bruner, JP May, JE McClure, M Steinberger, $H_{\infty}$ ring spectra and their applications, Lecture Notes in Mathematics 1176, Springer, Berlin (1986) MR836132

[7] R R Bruner, J Rognes, Differentials in the homological homotopy fixed point spectral sequence, Algebr. Geom. Topol. 5 (2005) 653-690 MR2153113

[8] F R Cohen, On configuration spaces, their homology, and Lie algebras, J. Pure Appl. Algebra 100 (1995) 19-42 MR1344842

[9] F R Cohen, T J Lada, J P May, The homology of iterated loop spaces, Springer, Berlin (1976) MR0436146

[10] Z Fiedorowicz, R M Vogt, Topological Hochschild Homology of $E_{n}-$ Ring Spectra, arXiv:math. AT/0410367

[11] PG Goerss, Associative MU algebras, preprint, available from http://www.math.nwu.edu/ pgoerss/

[12] P G Goerss, M J Hopkins, Moduli spaces of commutative ring spectra, from: "Structured ring spectra", London Math. Soc. Lecture Note Ser. 315, Cambridge Univ. Press, Cambridge (2004) 151-200 MR2125040

[13] M Hovey, N P Strickland, Morava K-theories and localisation, Mem. Amer. Math. Soc. 139 (1999) viii+100 MR1601906

[14] P Hu, I Kriz, JP May, Cores of spaces, spectra, and $E_{\infty}$ ring spectra, Homology Homotopy Appl. 3 (2001) 341-354 MR1856030 
[15] S O Kochman, Integral cohomology operations, from: "Current trends in algebraic topology, Part 1 (London, Ont., 1981)”, CMS Conf. Proc. 2, Amer. Math. Soc., Providence, R.I. (1982) 437-478 MR686130

[16] I Kriz, Towers of $E_{\infty}$-ring spectra with an application to BP, preprint (1995)

[17] A Lazarev, Towers of MU-algebras and the generalized Hopkins-Miller theorem, Proc. London Math. Soc. (3) 87 (2003) 498-522 MR1990937

[18] L G Lewis, The stable category and generalized Thom spectra, $\mathrm{PhD}$ thesis, University of Chicago (1978)

[19] L G Lewis, Jr, J P May, M Steinberger, , J E McClure, Equivariant stable homotopy theory, Lecture Notes in Mathematics 1213, Springer, Berlin (1986) MR866482

[20] J P May, $E_{\infty}$ ring spaces and $E_{\infty}$ ring spectra, Springer, Berlin (1977) MR0494077

[21] J E McClure, R E Staffeldt, On the topological Hochschild homology of bu. I, Amer. J. Math. 115 (1993) 1-45 MR1209233

[22] T Pirashvili, B Richter, Robinson-Whitehouse complex and stable homotopy, Topology 39 (2000) 525-530 MR1746906

[23] B Richter, A Robinson, Gamma homology of group algebras and of polynomial algebras, from: "Homotopy theory: relations with algebraic geometry, group cohomology, and algebraic $K$-theory", Contemp. Math. 346, Amer. Math. Soc., Providence, RI (2004) 453-461 MR2066509

[24] A Robinson, Gamma homology, Lie representations and $E_{\infty}$ multiplications, Invent. Math. 152 (2003) 331-348 MR1974890

[25] A Robinson, Classical obstructions and S-algebras, from: "Structured ring spectra", London Math. Soc. Lecture Note Ser. 315, Cambridge Univ. Press, Cambridge (2004) 133-149 MR2122157

[26] A Robinson, S Whitehouse, Operads and $\Gamma$-homology of commutative rings, Math. Proc. Cambridge Philos. Soc. 132 (2002) 197-234 MR1874215

[27] N P Strickland, Products on MU-modules, Trans. Amer. Math. Soc. 351 (1999) 25692606 MR1641115

[28] S Whitehouse, The integral tree representation of the symmetric group, J. Algebraic Combin. 13 (2001) 317-326 MR1836907

Fachbereich Mathematik der Universität Hamburg, Bundesstraße 55

20146 Hamburg, Germany

richter@math.uni-hamburg.de

http://www . math. uni-hamburg.de/home/richter/

Received: 25 May 2005 Revised: 17 November 2005

Algebraic $6 \mathcal{G}$ Geometric $\mathcal{T}$ opology, Volume 6 (2006) 